\title{
Epidemia do novo coronavírus (COVID-19): análise dos indicadores nos três primeiros meses, Estado de São Paulo, Brasil
}

New coronavirus epidemic (COVID-19): analysis of indicators in the first three months, state of São Paulo, Brazil Epidemia del nuevo coronavirus (COVID-19): análisis de indicadores en los primeros tres meses, estado de São Paulo, Brasil Roudom Ferreira MOURA ${ }^{1}$

Ana Paula Miranda MUNDIM-POMBO ${ }^{2}$

Rosângela Elaine Minéo BIAGOLINI ${ }^{3}$ Janessa de Fátima Morgado de OLIVEIRA ${ }^{4}$

'Doutorando em Ciências pelo departamento de Epidemiologia da FSPUSP. Enfermeiro da Divisão de Dengue, Chikungunya e Zika do Centro de Vigilância Epidemiológica "Prof. Alexandre Vranjac" (CVE) / Coordenadoria de Controle de Doenças (CCD) / Secretaria de Estado da Saúde de São Paulo (SESSP), Brasil. Professor da Universidade São Judas Tadeu. Professor do Curso de Pós-Graduação em Saúde Coletiva com ênfase em Saúde da Família da Universidade Santo Amaro (UNISA). Meste em Ciências - Faculdade de Saúde Pública da Universidade de São Paulo (FSPUSP). Especialização em Saúde Coletiva com enfoque no Programa de Saúde da Família - Escola de Enfermagem da

Universidade de São Paulo (EEUSP); Especialização em Educação em Saúde - Centro de Desenvolvimento em Saúde (CEDSS) da Universidade Federal de São Paulo (UNIFESP) e Especialização em Saúde da Mulher no Climatério (FSPUSP).

${ }^{2}$ Enfermeira e Médica Veterinária, Doutora em Ciências pela Faculdade de Medicina da Universidade de São Paulo, Professor da Universidade São Judas Tadeu - São Paulo-SP ${ }^{3}$ Enfermeira, Doutora em Ciências da Saúde pela Escola de Enfermagem da Universidade de São Paulo, Professora da Universidade Nove de Julho - São Paulo (SP). Enfermeira da Unidade de Vigilância em Saúde da Secretaria Municipal de Saúde de São Paulo-SP

${ }^{4}$ Farmacêutica-bioquímica, Doutora em Ciências pela Faculdade de Saúde Pública da Universidade de São Paulo, Investigadora - Departamento de Epidemiologia do Instituto de Saúde Pública da Universidade do Porto (Projeto DOCnet). Porto, Portugal

\section{Resumo}

Introdução: O Estado de São Paulo foi a Unidade da Federação onde ocorreu a notificação do primeiro caso de COVID-19 no Brasil e América Latina' apresentando indicadores de saúde alarmantes e boa parte dos municípios afetados. Objetivo: Analisar os indicadores de saúde do Novo Coronavírus (COVID-19) no Estado de São Paulo (ESP) nos três primeiros meses da epidemia a partir da confirmação do primeiro caso. Material e método: Estudo ecológico, descritivo, considerando os casos confirmados de COVID-19 captados pelo Centro de Vigilância Epidemiológica do ESP para o período de 26 de fevereiro a 26 de maio de 2020. Resultados: Houve crescimento do número de municípios (35, 284 e 510), casos confirmados (1.015, 20.652 e 85.459), óbitos (57, 1.700 e 6.423), coeficientes de incidência (2,21; 44,97 e 186,11 para cada 100.000 habitantes) e mortalidade $(0,12 ; 3,70$ e 13,99 para cada 100.000 habitantes) - respectivamente, março, abril e maio. Observou-se declínio do coeficiente de letalidade no terceiro mês comparado ao segundo (respectivamente, 8,23 e 7,52 para cada 100 casos). Conclusão: A magnitude do COVID-19 extrapola os indicadores mundiais em algumas localidades do Estado de São Paulo.

Descritores: Infecções por Coronavírus; Pandemias; Epidemiologia Descritiva; Estudos Ecológicos.

\section{Abstract}

Introduction: The state of São Paulo was the Federation Unit where the first case of COVID-19 was reported in Brazil and Latin America, presenting alarming health indicators and most of the municipalities affected. Objetive: to analyze the health indicators of the New Coronavirus (COVID-19) in the State of São Paulo (ESP) in the first three months of the epidemic after the confirmation of the first case. Material and methods: Ecological, descriptive study, taking into account the confirmed cases of COVID-19 captured by the Epidemiological Surveillance Center of São Paulo State from February 26 to May 26, 2020. Results: There was an increase in relation to the number of municipalities (35, 284 and 510), confirmed cases $(1,015,20,652$ and 85,459), deaths (57, 1,700 and 6,423), incidence coefficients (2.21; 44.97 and 186.11 per 100,000 inhabitants) and mortality (0.12; 3.70 and 13.99 per 100,000 inhabitants) - respectively for March, April and May. However, the decline in the lethality rate in the third month $(7.52 \%)$ is observed in comparison to the second month $(8.23 \%)$. Conclusion: The magnitude of COVID-19 indicators showed higher than the world indicators in some municipalities.

Descriptors: Coronavirus Infections; Pandemics; Epidemiology, Descriptive; Ecological Studies.

\section{Resumen}

Introdución: El estado de São Paulo fue la Unidad de la Federación donde se notificó el primer caso de COVID-19 en Brasil y América Latina, presentando indicadores de salud alarmantes y la mayoría de los municipios afectados. Objetivo: Analizar los indicadores de salud del nuevo coronavirus (COVID-19) en el estado de São Paulo (ESP) en los primeros tres meses de la epidemia después de la confirmación del primer caso. Material y método: Estudio descriptivo ecológico, considerando los casos confirmados de COVID-19 capturados por el Centro de Vigilancia Epidemiológica ESP para el período del 26 de febrero al 26 de mayo de 2020. Resultados: Hubo un aumento en el número de municipios (35, 284 y 510), casos confirmados $(1,015,20,652$ y 85,459), muertes $(57,1,700$ y 6,423$)$, coeficientes de incidencia (2.21; 44.97 y 186.11 por cada 100,000 habitantes) y mortalidad $(0.12 ; 3,70$ y 13,99 por cada 100.000 habitantes): marzo, abril y mayo, respectivamente. Hubo una disminución en el coeficiente de letalidad en el tercer mes en comparación con el segundo (respectivamente, 8.23 y 7.52 por cada 100 casos). Conclusión: La magnitud de COVID-19 va más allá de los indicadores globales en algunos lugares del estado de São Paulo.

Descriptores: infecciones por Coronavirus; Pandemias; Epidemiología Descriptiva; Estudios Ecológicos.

\section{INTRODUÇÃO}

Após investigação de surto de pneumonia viral de origem desconhecida no município de Wuhan, província de Hubei, China, em dezembro de 2019 , foi isolado em janeiro de 2020, um novo vírus extremamente contagioso, o SARS-CoV-2 (Severe Acute Respiratory Syndrome 2) ${ }^{1-3}$.

O SARS-CoV-2, causador da Síndrome Respiratória Aguda Grave (SRAG), Novo Coronavírus (COVID-19) 1,3, se disseminou intensamente desde o primeiro caso confirmado, de modo que em janeiro de 2020, a Organização Mundial da Saúde (OMS) declarou o surto como emergência de saúde pública de interesse internacional, que devido expansão em nível global, em março de 2020, foi declarado como pandemia ${ }^{3-5}$.

A disseminação do SARS-CoV-2 avançou de modo que em 06 de junho de 2020 já haviam sido confirmados 6.663.304 casos e 392.802 mortes de COVID-19 no mundo. Essa casuística foi, principalmente, composta por notificações oriundas das regiões: Americana (3.155.370 casos/ 176.167 mortes), Europeia (2.249.999 casos/ 183.128 mortes), Mediterrâneo oriental (605.026 casos/ 14.024 mortes), Pacífico Ocidental (189.030 casos/ 7.092 mortes), Sudeste asiático (336.577 casos/ 9.316 mortes) e Africana (126.561 casos/ 3.062 mortes) ${ }^{6}$.

Nas Américas chamou atenção a casuística apresentada pelo Brasil, uma vez que, em 23 de maio 
de 2020, já haviam sido confirmados 347.398 casos e 22.013 mortes, correspondendo a 6,3\% dos casos ${ }^{7}$, sendo assim, o Brasil figurava nessa data como o principal país acometido pela COVID-19 na América Latina, estando em segundo lugar no ranking mundial ${ }^{6}$.

Os casos e óbitos no Brasil, monitorados continuamente pelas Unidades de Vigilância Epidemiológica, durante o período a que se refere o presente estudo, se apresentam de forma crescente e exponencial. Além disso, não haviam sido observados sinais de desaceleração desse crescimento, no período estudado, cenário identificado em todas as Unidades Federativas do país ${ }^{7}$.

O Brasil vive um contexto onde nem sempre as recomendações de distanciamento físico e higienização são possíveis de seguir em localidades com submoradias, onde, geralmente, vivem muitas pessoas por cômodos e/ou em áreas onde o acesso à água limpa é difícil, ou, onde mesmo a necessidade do emprego informal impede de tomar as medidas profiláticas cabíveis ${ }^{8}$.

Entre as principais regiões do país acometidas pela COVID-19, estão as Norte e Sudeste $^{7}$. Destaca-se na região Sudeste o Estado de São Paulo, onde ocorreu a notificação do primeiro caso no Brasil e América Latina ${ }^{9}$, e que, por sua vez, apresenta-se como principal Unidade da Federação em termos de municípios afetados (504 cidades entre as 645 do estado) ${ }^{7}$.

Compreender a epidemiologia da COVID-19 fundamenta $o$ interesse em investigar mais detalhadamente esse problema de saúde pública no Estado de São Paulo, na expectativa de contribuir, gerando subsídios aos gestores para decisões em saúde pública. Diante do exposto, o presente estudo teve como objetivo descrever os indicadores de saúde do COVID-19, de 26 de fevereiro a 26 de maio de 2020, no Estado de São Paulo.

\section{MATERIAL E MÉTODO}

Estudo epidemiológico, do tipo ecológico, de base populacional. A população do Estado de São Paulo foi objeto do estudo, considerando sua distribuição nos 645 municípios do estado. Os casos confirmados de COVID-19 foram obtidos a partir da base de dados de domínio público do Centro de Vigilância Epidemiológica "Prof. Alexandre Vranjac" / Centro de Controle de Doenças / Secretaria de Estado da Saúde / Governo do Estado de São Paulo ${ }^{10}$. Para o cálculo dos coeficientes de incidência e mortalidade, foi utilizada a população em 2019 obtida do sítio eletrônico do Instituto Brasileiro de Geografia e Estatística (IBGE) ${ }^{11}$. Os coeficientes de incidência e mortalidade por 100.000 habitantes foram calculados a partir da razão entre o número de casos confirmados e óbitos, respectivamente (numerador) e a população residente (denominador). O coeficiente de letalidade por 100 casos foi calculado a partir da razão entre o número de óbitos (numerador) e os casos confirmados (denominador). Para a análise dos dados, utilizou-se estatística descritiva, aplicando-se os Softwares TabWin 3.6b, Microsoft Excel 2016. Para confecção dos mapas foi utilizado o software SIGs ArcGis versão 10.5 e Qgis10. A pesquisa não precisou ser aprovada por Comitê de Ética de Pesquisa, haja vista que os dados secundários da COVID-19 obtidos para análise nesse estudo são de domínio público e não apresentou identificação dos pacientes, seguindo, assim, os princípios estabelecidos pela Resolução ${ }^{\circ}$ 466, de 12 de dezembro de 2012, que dispõe sobre as diretrizes e normas regulamentadoras de pesquisa envolvendo seres humanos.

\section{RESULTADOS}

O primeiro caso de COVID-19 no Estado de São Paulo ocorreu na capital e foi publicado em 26 de fevereiro de 2020 pelo Centro de Vigilância Epidemiológica "Prof. Alexandre Vranjac" / Centro de Controle de Doenças / Secretaria de Estado da Saúde / Governo do Estado de São Paulo ${ }^{10}$. Após três meses da epidemia por COVID-19 (26 de maio de 2020), o Estado de São Paulo já tinha acumulado 85.459 casos confirmados em 510 municípios (79,07\% dos 645 municípios), sendo o município de São Paulo o mais atingindo (55,64\%) (Figura 1).

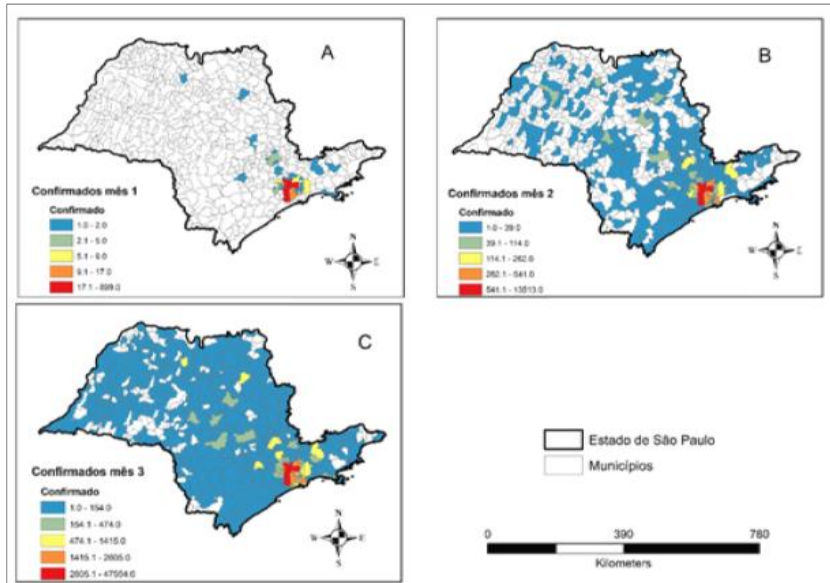

Figura 1: Distribuição dos casos confirmados do Novo Coronavírus (2019-nCoV) (A-1 mês após o primeiro caso confirmado; $\mathbf{B}-2$ meses após o primeiro caso e $\mathbf{C}$ - 3 meses após o primeiro caso), segundo município de residência. Estado de São Paulo, 26 de fevereiro a 26 de maio de 2020 (Fonte: Centro de Vigilância Epidemiológica "Prof. Alexandre Vranjac" / Centro de Controle de Doenças / Secretaria de Estado da Saúde / Governo do Estado de São Paulo ${ }^{10}$ ).

Houve variação de $1.934,68 \%$ no número de casos confirmados do primeiro (1.015 casos) para o segundo (20.652 casos) e $8.319,61 \%$ do primeiro para o terceiro mês (85.459 casos), respectivamente. Para o período estudado, o Estado de São Paulo apresentou o coeficiente de incidência acumulado de COVID-19 de 186,11 casos para cada 100.000 habitantes. Os municípios com as maiores estimativas de risco foram Jaci $(1089,57$ casos para cada 100.000 habitantes), Santos $(601,18$ casos para cada 100.000 
habitantes), Santa Salete (582,52 casos para cada 100.000 habitantes), Pariquera-Açu (468,24 casos para cada 100.000 habitantes) e São Paulo $(388,13$ casos para cada 100.000 habitantes) (Figura 2).

O primeiro óbito por COVID-19 foi registrado em 17 de março de 2020 no município de São Paulo e em 26 de maio o Estado de São Paulo confirmou 6.423 óbitos em 224 municípios $(37,83 \%$ dos 645 municípios). O município de São Paulo obteve o maior número de óbitos $(57,47 \%)$ para o período. A variação dos óbitos entre os meses de março (35 óbitos) e abril (284 óbitos) foi de $711,43 \%$. Entre os meses de março e maio (6.423 casos) foi de $18.251,43 \%$ (Figura 3 ).

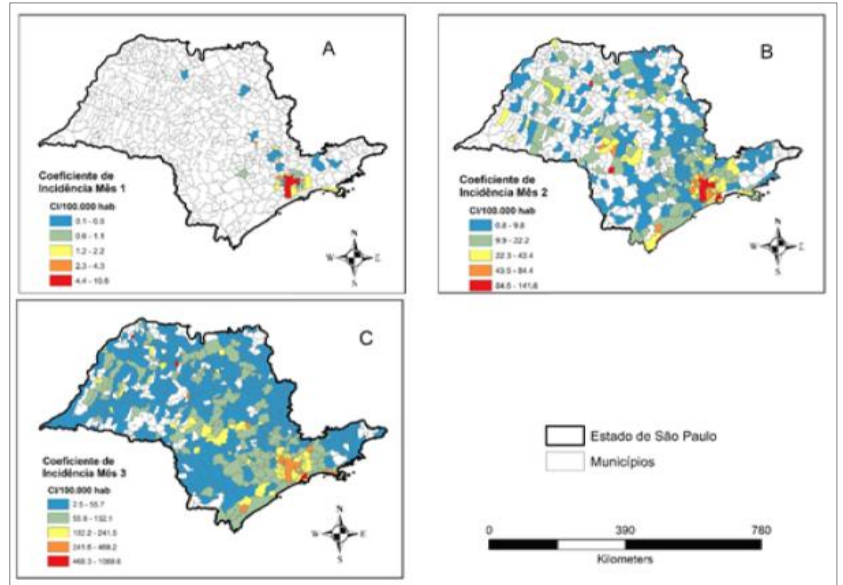

Figura 2 - Distribuição do coeficiente de incidência (por 100.000 habitantes) do Novo Coronavírus (2019-nCoV) (A - 1 mês após o primeiro caso confirmado; $\mathbf{B}-2$ meses após o primeiro caso e $\mathbf{C}-3$ meses após o primeiro caso confirmado), segundo município de residência. Estado de São Paulo, 26 de fevereiro a 26 de maio de 2020 (Fonte: Centro de Vigilância Epidemiológica "Prof. Alexandre Vranjac" / Centro de Controle de Doenças / Secretaria de Estado da Saúde / Governo do Estado de São Paulo ${ }^{10}$ )

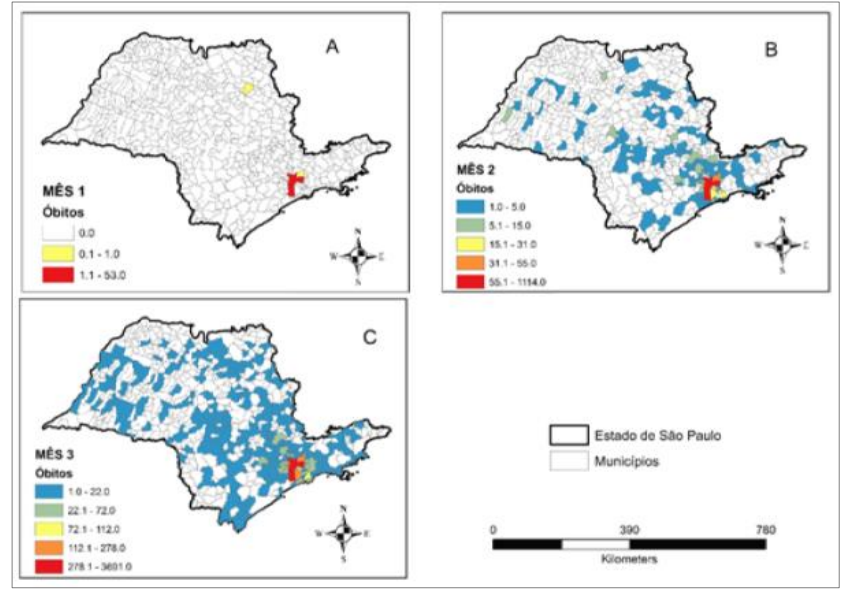

Figura 3 - Distribuição dos óbitos do Novo Coronavírus (2019-nCoV) (A - 1 mês após o primeiro caso confirmado; $\mathbf{B}-2$ meses após o primeiro caso e $\mathbf{C}-3$ meses após o primeiro caso confirmado), segundo município de residência. Estado de São Paulo, 26 de fevereiro a 26 de maio de 2020 (Fonte: Centro de Vigilância Epidemiológica "Prof. Alexandre Vranjac" / Centro de Controle de Doenças / Secretaria de Estado da Saúde / Governo do Estado de São Paulo ${ }^{10}$ ).

O coeficiente de mortalidade de COVID-19 acumulado para os três meses no Estado de São Paulo foi de 13,99 casos, sendo os municípios de maiores estimativas para esse indicador: Uru - 85,84; Monções - 44,27; Torre de Pedra - 41,46; Osasco 39,80; São João das Duas Pontes - 38,94; Barueri 37,57; Ipiguá - 37,09; Pedrinhas Paulista - 32,33;
Arandu - 31,46 e São Paulo-30,13, todos para cada 100.000 habitantes, respectivamente (Figura 4).

A razão entre o número de óbitos e de casos confirmados (letalidade) acumulada para os três meses por COVID-19 no Estado de São Paulo foi de 7,52 para cada 100 casos. Os municípios de Alto Alegre, Álvaro de Carvalho, Bofete, Caiabu, Guará, Iepê, Pitangueiras, Poloni, Pradópolis, Santo Antônio da Alegria e São Luís do Paraitinga apresentaram 100\% de letalidade por COVID-19 (Figura 5).

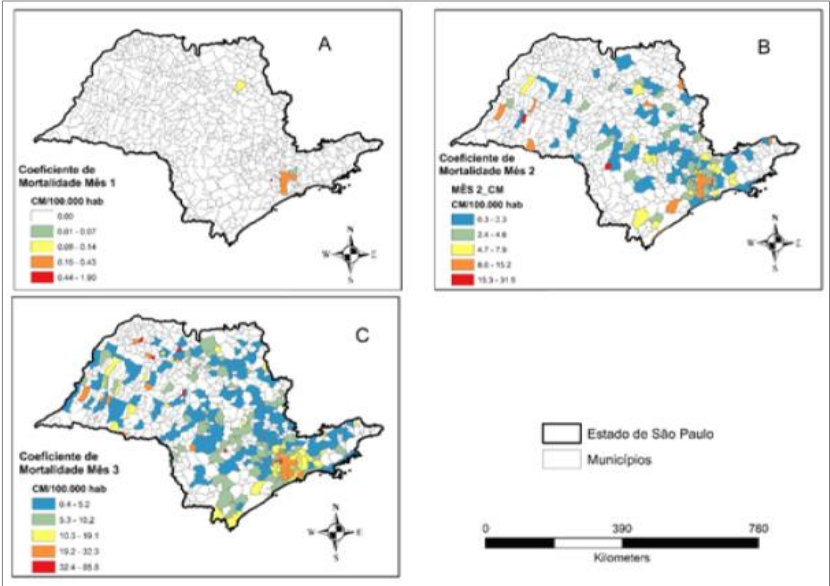

Figura 4 - Distribuição do coeficiente de mortalidade (por 100.000 habitantes) do Novo Coronavírus (2019-nCoV) (A - 1 mês após o primeiro caso confirmado; $\mathbf{B}$ - 2 meses após o primeiro caso e $\mathbf{C}-3$ meses após o primeiro caso confirmado), segundo município de residência. Estado de São Paulo, 26 de fevereiro a 26 de maio de 2020 (Fonte: Centro de Vigilância Epidemiológica "Prof. Alexandre Vranjac" / Centro de Controle de Doenças / Secretaria de Estado da Saúde / Governo do Estado de São Paulo ${ }^{10}$ ).

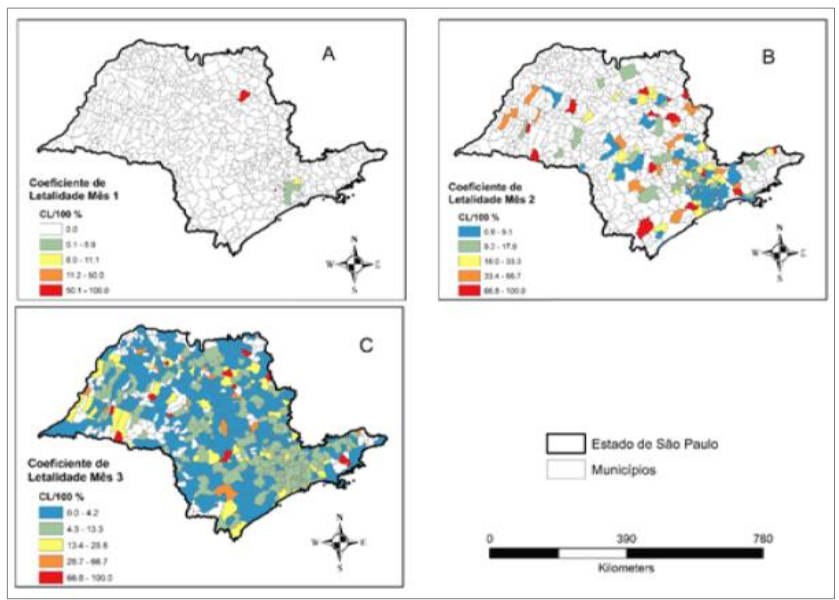

Figura 5 - Distribuição do coeficiente de letalidade do Novo Coronavírus (2019$\mathrm{nCoV})$ (A - 1 mês após o primeiro caso confirmado; $\mathbf{B}-2$ meses após o primeiro caso e $\mathbf{C}-3$ meses após o primeiro caso confirmado), segundo município de residência. Estado de São Paulo, 26 de fevereiro a 26 de maio de 2020 (Fonte: Centro de Vigilância Epidemiológica "Prof. Alexandre Vranjac" / Centro de Controle de Doenças / Secretaria de Estado da Saúde / Governo do Estado de São Paulo ${ }^{10}$ ).

\section{DISCUSSÃO}

Após a transição epidemiológica, grande atenção tem sido concentrada sobre os impactos das doenças não transmissíveis sobre a saúde humana. Entretanto, desde a década de 80 tem havido um aumento do número de epidemias e uma parte delas se deve a enfermidades emergentes, o que pode em parte ser atribuído a agentes que sofreram mudanças evolutivas recentes ${ }^{12}$.

A relevância das doenças emergentes e 
reemergentes fez com que, a partir de 2005, a OMS tenha modificado o Regulamento Sanitário, estabelecendo, entre outros, a situação epidemiológica denominada Emergência de Saúde Pública de Importância Internacional (ESPII), que é definida como um evento extraordinário que constitui um risco para a saúde pública, devido à propagação internacional de uma enfermidade e que pode requerer uma resposta internacional coordenada. A ESPII desde 2005 foi declarada em seis ocasiões, sendo a última delas devido à atual pandemia ${ }^{12}$.

A atual pandemia por COVID-19 colocou o Brasil como um país com destaque para o número de infectados e de mortos, com destaque para o Estado de São Paulo. Frente à impossibilidade de redução de pessoas susceptíveis por imunização, uma vez que não há vacina para a doença, a redução da velocidade de crescimento da curva epidêmica fica condicionada ao sucesso das ações de isolamento físico social. Além disso, tem impacto positivo sobre o controle da doença, a identificação precoce dos casos positivos, com a adoção de ações de isolamento tanto domiciliar quanto hospitalar e, consequentemente, a redução nos indicadores da doença e de aumento no tempo de duplicação ${ }^{2}$.

Assim, o impacto da epidemia no Estado de São Paulo tem relação com a resposta da população, influenciada, em parte, pela ausência de padronização nas orientações/medidas adotadas nos níveis municipal, estadual e federal, bem como pela baixa testagem e pelas características relacionadas às desigualdades socioeconômicas/estrutura social próprias das regiões, representando um desafio, com seus impactos na capacidade de resposta do sistema de saúde e de curto e médio prazo na economia ${ }^{12}$.

$\mathrm{O}$ presente estudo, ao analisar a epidemia de COVID-19 no Estado de São Paulo, identificou que os coeficientes de incidência e de mortalidade para o Estado de São Paulo, para o período estudado, foram 186,1 e 14,0 para 100.000 habitantes, respectivamente, e para a capital foram 388,1 e 30,1 para 100.000 habitantes, respectivamente, bem mais altos que os observados em nível mundial. Considerando todos os casos desde o início da pandemia até o dia 7 de junho, o coeficiente de incidência foi 90,0 casos para 100.000 habitantes e a de mortalidade foi de 5,2 óbitos para cada 100.000 habitantes ${ }^{13}$. A tendência mundial para o coeficiente de incidência se apresenta crescente no período proposto deste estudo e para a mortalidade está decrescente a partir de meados de abril, no entando, para a capital e para o Estado de São Paulo, mantémse crescente, como observado pela variação de magnitude dos indicadores.

Devido à grande concentração populacional $\mathrm{e}$ às condições socioeconômicas mais díspares, aliadas ao fato de receberem muitos viajantes de várias partes do país e do mundo, por sua importância econômica, e apresentarem regiões com grande população flutuante, as megacidades como São Paulo foram confrontadas com tensões maiores dos surtos e importância na disseminação da infecção por COVID- $19^{14}$.

Os municípios próximos à capital, também, apresentaram incidência e mortalidade altos, padrão que não foi observado para a letalidade. A movimentação da população entre as cidades integrantes da Grande São Paulo e os municípios vizinhos favorece a disseminação do agente ${ }^{12}$. Assim, assim regiões próximas geograficamente tendem a apresentar coeficientes mais semelhantes do que as mais afastadas ${ }^{15}$.

Há diferenças importantes entre os municípios que apresentaram as maiores magnitudes para cada indicador estudado. Os municípios com maiores coeficientes de incidência foram Jaci, que mantém a magnitude para o indicador maior que o da capital do estado desde a avaliação feita para o segundo mês de epidemia, Santos e Pariquera-Açu, que superaram a magnitude da capital paulista no último mês estudado. Os maiores coeficientes de mortalidade foram observados para Uru, Monções, Torre de Pedra, Osasco, Osasco, São João das Duas Pontes, Barueri, Pedrinhas Paulistas - cuja magnitude superou a observada para a capital do estado no terceiro mês da epidemia - e Arandu - que manteve a magnitude superior à da capital para o indicador.

De acordo com Organização Pan-americana de Saúde (OPAS) ${ }^{16}$, o coeficiente de mortalidade específico por doenças transmissíveis estima o risco de morte pela doença considerada na população total da área geográfica em questão em um determinado período de tempo, refletindo a efetividade de medidas de prevenção e controle, bem como as condições de diagnóstico e da assistência médica oferecida à população. Levando isso em conta, salienta-se a necessidade de adoção de medidas de mitigação do contágio mais efetivas em Jaci e de identificação dos doentes graves e tratamento em tempo hábil para o município de Arandu, que contava apenas com dois estabelecimentos de saúde do Sistema Único de Saúde (SUS) e 20 leitos para internação em $2009^{11}$. No entanto, o fato de a população do município ser pequena pode justificar a alta mortalidade observada para o município.

A letalidade observada no período de estudo foi de $100 \%$ em Alto Alegre, Álvaro de Carvalho, Bofete, Guará, Poloni, Pradópolis e São Luís do Paraitinga, onde não havia casos até 26 de abril, e se manteve assim desde o segundo mês da epidemia em Caiabu, Iepê, Pitangueiras e Santo Antônio da Alegria. A hipótese para justificar este achado é que o número de casos da doença tenha sido muito pequeno e que os poucos casos tenham levado a óbito - o que pode justificar a letalidade de $100 \%$ em 11 municípios do estado. 
De acordo com os dados do World-o-Meter ${ }^{13}$ para o dia 7 de junho, a letalidade da doença no mundo foi $5,7 \%$ (7.013.552 casos confirmados no mundo e 402.744 óbitos), tendo decrescido desde 14 de maio, quando era $6,7 \%$. Acredita-se que o fato da letalidade ser alta nesses municípios, deve-se a subestimação dos casos, pois, segundo publicação recente, são necessárias evidências sólidas (de boa qualidade) para combater as iniquidades em saúde durante o surto de COVID-19. Sem elas, a avaliação da equidade em saúde será impossível, o que reduzirá o impacto dos esforços de controle da doença. Além disso, garantir oportunidades de diagnóstico e tratamento para todos é visto como um fator importante para o enfrentamento e superação da doença ${ }^{17}$.

Por outro lado, em nove municípios que apresentaram mais de 200 casos confirmados da doença, a letalidade ficou abaixo da observada para o mundo (5,7\%)12: Bauru (4,7\%; 253 casos), Botucatu $(3,2 \% ; 218$ casos), Campinas (4,3\%; 1.415 casos), Ribeirão Preto (3,1\%; 652 casos); Santana de Parnaíba (3,2\%; 404 casos), Santos $(4,3 \% ; 2.605$ casos), São José do Rio Preto (3,5\%; 568 casos), São José dos Campos (4,3\%; 694 casos) e São Sebastião $(0,8 \% ; 263$ casos). Ressalta-se que há um hospital universitário, ou, hospital-escola em seis desses municípios com letalidade abaixo da observada mundialmente (Bauru, Botucatu, Campinas, Ribeirão Preto, Santos e São José do Rio Preto).

Os municípios de Bauru (1,76), Botucatu $(3,87)$, São José do Rio Preto $(1,99)$, Santos $(2,01)$ e Ribeirão Preto $(2,05)$ apresentavam um número de leitos disponíveis do SUS para 1.000 habitantes superior ao observado para o Estado de São Paulo (1,18 leitos), considerando os dados disponíveis para $2019^{18}$. Deve-se considerar com cuidado os dados sobre a disponibilidade de leitos, pois não consideram os hospitais de campanha que foram montados em alguns municípios.

Três municípios que apresentaram mais de 200 casos da doença possuem uma proporção de população idosa (com idade igual ou superior a 60 anos) inferior aos 15,3\% observados para o estado (Santana do Parnaíba - 12,0\%; São José dos Campos - 14,6\%; e São Sebastião - 11,5\%), considerando as estimativas disponíveis para $2020^{18}$.

O Boletim Completo da Fundação Sistema Estadual de Análise de Dados (SEADE) do Estado de São Paulo Contra o Coronavírus apresentou informação sobre a adesão ao isolamento social, em 8 de junho de 2020, e São Sebastião era o município com o melhor indicador, com $58 \%$ de adesão ${ }^{19}$.

A baixa letalidade observada para esses nove municípios pode ser justificada pelos fatores acima descritos, ao menos em partes, pois é conhecido que eles podem impactar tanto na suscetibilidade ao agravamento da doença, quanto na disponibilidade $\mathrm{e}$ acesso ao tratamento adequado.

O presente estudo é ecológico descritivo e comparativo dos indicadores incidência, mortalidade e letalidade para os três primeiros meses da epidemia no estado. A maior limitação da análise de dados ecológicos conduzida no presente estudo tem relação com a heterogeneidade entre os municípios e populações no nível de exposição à doença ${ }^{15} \mathrm{e}$ aos fatores que influenciam seu impacto como problema de saúde pública, como a estrutura etária da população, a extensão da investigação de casos, o acesso em tempo oportuno a serviços de saúde, adoção de medidas de isolamento social e de outras medidas de redução do risco de contágio não serem conhecidos. Tais fatores não foram incluídos nas análises.

Além disso, conduzir a comparação simples dos indicadores entre as regiões estudadas pode ser complexo, pois regiões com número de casos observados pequenos mostram grande variabilidade para os coeficientes estimados ${ }^{16}$. Assim, os coeficientes mais altos tendem a ser observados para municípios pequenos, como pôde ser observado, provavelmente, devido aos clusters de casos.

CONCLUSÃO

A análise espacial dos indicadores de saúde do COVID-19 nos três primeiros meses da epidemia possibilita entender a distribuição desigual da doença e dos óbitos no Estado de São Paulo.

Não obstante ao fato que a falta da testagem prejudica a análise dos coeficientes de incidência da doença, observa-se que o aumento dos casos indica, potencialmente, a infectividade da doença, e pode-se inferir a partir delas os municípios com maior disseminação da doença.

A distribuição dos municípios com maiores coeficientes de mortalidade no Estado de São Paulo aponta para a necessidade de ampliar a análise para esses locais, identificando-se a composição da população, segundo faixas etárias, pois o coeficiente pode estar associado a estrutura etária da população.

A identificação dos municípios que tiveram baixa letalidade, mesmo com incidência alta, e a investigação de questões relativas à assistência à saúde quanto, por exemplo, ao tratamento precoce dos casos graves e a oferta de leitos de Unidade de Terapia Intensiva (UTI), pode ajudar a justificar estes achados.

\section{REFERÊNCIAS}

1. Tan W, Zhao X, Ma X, Wang W, Niu P, Xu W et al. A Novel Coronavirus Genome Identified in a Cluster of Pneumonia Cases - Wuhan, China 2019-2020. China CDC Weekly, 2020;2(4):61-2.

2. Rafael RDMR, Neto M, Carvalho MMB de, David HMSL, Acioli S, Faria MG de A. Epidemiologia, políticas públicas e pandemia de Covid-19: o que esperar no Brasil? Rev enferm 
UERJ. 2020;28:e49570.

3. Wang C, Horby PW, Hayden FG, Gao GF. A novel coronavirus outbreak of global health concern [published correction appears in Lancet. 2020. Lancet. 2020;395(10223):470-73.

4. Di Gennaro F, Pizzol D, Marotta C, Antunes M, Racalbuto $\mathrm{V}$, Veronese $\mathrm{N}$ et al. Coronavirus Diseases (COVID-19) Current Status and Future Perspectives: A Narrative Review. Int J Environ Res Public Health. 2020;17(8):2690.

5. World Health Organization. Coronavirus disease (COVID-19). Coronavirus disease (COVID-2019) Situation Report - 117. World Heal Organ [Internet]. 2020;8(1):3-8. Available at: https://www.who.int/docs/defaultsource/coronaviruse/situation-reports/20200516covid-19-sitrep-117.pdf?sfvrsn=8f562cc

6. World Health Organization. Coronavirus disease (COVID-19). Coronavirus disease (COVID-19) Situation Report 138. 2020;(June). Available at: https://www.who.int/docs/defaultsource/coronaviruse/situation-reports/20200606covid-19-sitrep-138.pdf?sfvrsn=c8abfb17_4

7. BRASIL. Ministério da Saúde $S$ de $\mathrm{V}$ em S. Boletim Epidemiológico Especial COE COVID19. Bol Epidemiológico Espec COE-COVID19 [Internet]. 2020; Available at: https://www.saude.gov.br/images/pdf/2020/May/2 9/2020-05-25---BEE17---Boletim-do-COE.pdf.

8. The Lancet. COVID-19 in Brazil: "So what?". Lancet. 2020;395(10235):1461.

9. Rodriguez-Morales AJ, Gallego V, EscaleraAntezana JP, Mendez CA, Zambrano LI, FrancoParedes et. al. COVID-19 in Latin America: The implications of the first confirmed case in Brazil. Travel Med Infect Dis. 2020;35:101613.

10. Governo do Estado de São Paulo10 - Secretaria de Estado da Saúde - Coordenadoria de Controle de Doenças - Centro de Vigilância Epidemiológica "Prof. Alexandre Vranjac" - Novo Coronavírus (COVID-19) - Situação Epidemiológica 26 de fevereiro a 26 de maio de 2020, disponíveis em: http://www.saude.sp.gov.br/cve-centro-devigilancia-epidemiologica-prof.-alexandrevranjac/areas-de-vigilancia/doencas-detransmissao-respiratoria/coronavirus-covid19/situacao-epidemiologica

11. IBGE. Instituto Brasileiro de Geografia e Estatística. Brasil. São Paulo. Arandu. Disponível em:

https://cidades.ibge.gov.br/brasil/sp/arandu/pesqui $\mathrm{sa} / 37 / 30255$

12. Castro Delgado R, Arcos González P. Analyzing the health system's capacity to respond to epidemics: a key element in planning for emergencies. El análisis de la capacidad de respuesta sanitaria como elemento clave en la planificación ante emergencias epidémicas. Emergencias. 2020;32(3):157-59.

13. World-o-Meter. Disponível em: https://www.worldometers.info/coronavirus/. Acesso em: 14 de maio de 2020.

14. Ren H, Zhao L, Zhang A, Song L, Liao Y, Lu W et al. Early forecasting of the potential risk zones of COVID-19 in China's megacities. Sci Total Environ. 2020;729:138995.

15. Morgenstern H. Ecologic studies in epidemiology: concepts, principles, and methods. Annu Rev Public Health. 1995;16:61-81.

16. Organização Pan-americana de Saúde. REDE Interagencial de Informação para a Saúde RIPSA. Indicadores Básicos para a Saúde no Brasil: conceitos e aplicações. 2. ed. - Brasília: Organização Pan-Americana da Saúde, 2008. p. $144 . \quad$ Disponível em: http://tabnet.datasus.gov.br/tabdata/livroidb/2ed/C apituloC.pdf

17. Medeiros de Figueiredo A, Daponte A, Moreira Marculino de Figueiredo DC, Gil-García E, Kalache A. Letalidad del COVID-19: ausencia de patrón epidemiológico [Case fatality rate of COVID-19: absence of epidemiological pattern] Gac Sanit. 2020;S0213-9111(20)30084-4.

18. SEADE - Fundação Sistema Estadual de Análise de Dados. Perfil dos Municípios Paulistas. Disponível em: https://perfil.seade.gov.br/. Acessado em: 10/06/2020.

19. Governo de São Paulo. SP Contra o Novo Coronavírus. Adesão ao Isolamento Social em São Paulo. Disponível em: https://www.saopaulo.sp.gov.br/coronavirus/isola mento/. Acessado em 10/06/2020.

\section{CONFLITO DE INTERESSES}

Os autores declaram não haver conflitos de interesse.

\section{AUTOR PARA CORRESPONDÊNCIA}

\section{Roudom Ferreira Moura}

Avenida Celso Garcia, 4061 - Apto. 21, Tatuapé

03063-000 São Paulo - SP, Brasil

Telefone - (55)11.98047-7239

e-mail:rfmoura@saude.sp.gov.br
Submetido em 08/07/2020 Aceito em 24/08/2020 